\title{
THE EIGENVALUE BEHAVIOUR FOR THE BOUNDARY VALUE PROBLEMS RELATED TO SELF-SIMILAR MEASURES ON $\mathbb{R}^{d}$
}

\author{
K. NAimark And M. Solomyak
}

\section{Introduction}

Suppose that a compactly supported probability measure $\mu$ on $\mathbb{R}^{d}$ is given. In a domain $X \subset \mathbb{R}^{d}$ such that Supp $\mu \subset \bar{X}$, we consider spectral problems whose "weak" description is as follows: let $\mathcal{V}=\stackrel{\circ}{L}_{2}^{1}(X)$ or $\mathcal{V}=$ $\left\{v \in L_{2}^{1}(X): \int_{\bar{X}} v d \mu=0\right\}$. The spaces $\stackrel{\circ}{L}_{2}^{1}(X)$ and $L_{2}^{1}(X)$ are closely related to the Sobolev spaces $H_{0}^{1}(X)$ and $H^{1}(X)$ respectively and coincide with them if $X$ is, say, a bounded domain with the sufficiently smooth boundary. Definitions and some details about these spaces are given in Section 3 .

We seek the eigenvalues $\lambda$ and eigenfunctions $u \in \mathcal{V}$ of the equation

$$
\lambda \int_{X} \nabla u \cdot \nabla \bar{v} d x=\int_{\bar{X}} u \bar{v} d \mu, \quad \text { any } v \in \mathcal{V}
$$

see Section 4 for an accurate statement of the problem. If $\mu$ is absolutely continuous, $d \mu=V d x$, then (1) corresponds to the classical boundary value problem

$$
-\lambda \Delta u=V u,
$$

with the Dirichlet or Neumann conditions on $\partial X$ respectively.

Suppose in addition that the operator associated with the problem (1) is compact. Then its nonzero spectrum consists of a sequence of positive eigenvalues $\lambda_{k}, \quad \lambda_{k} \rightarrow 0$. Let $n(t)$ be the corresponding distribution function:

$$
n(t)=\#\left\{k: \lambda_{k}>t\right\}, \quad t>0 .
$$

Received November 17, 1994.

The second author was supported by a grant from the Minerva Foundation. 
We are interested in the behaviour of $n(t)$ when $t \rightarrow 0$. This behaviour is well known for absolutely continuous measures, when we are dealing with the equation (2). Namely, under some, rather mild assumptions on $V$ (and, for the Neumann boundary value problem, also on $\partial X$ ), one has

$$
n(t)=O\left(t^{-\frac{d}{2}}\right)
$$

Moreover, $n(t)$ has a regular asymptotic behaviour at 0 :

$$
\lim _{t \rightarrow 0} t^{\frac{d}{2}} n(t)=\frac{(4 \pi)^{-\frac{d}{2}}}{\Gamma\left(\frac{d}{2}+1\right)} \int_{X} V^{\frac{d}{2}} d x
$$

see [BS] for proofs and a detailed discussion of (3) and (4).

Much less is known for singular measures $\mu$. In this case (1) can not in general be rewritten as a classical boundary value problem. Notice only that (1) implies $\Delta u(x)=0$ outside Supp $\mu$. In this paper we consider the case of self-similar measures $\mu$ which constitute an important subclass of general singular measures. For such a $\mu$, we show that $n(t)$ has a powerlike growth at $t=0$ :

$$
c t^{-\delta} \leq n(t) \leq C t^{-\delta}, \quad t \leq t_{0} .
$$

We give an expression for $\delta$ in (5) and discuss the relations between $\delta$ and the Hausdorff dimensions of the measure $\mu$ and of its support.

Spectral problems related to fractals have been rather popular during the last several years; e.g., see the papers by Lapidus [La] and by Levitin and Vassiliev [LeVa], which include, in particular, a survey of the topic. The reference lists in these two papers together cover most of the literature devoted to this class of problems.

However, the problems investigated in all these works are essentially different from the one considered here. The only exception known to us is the paper $[\mathrm{Fu}]$ by Fujita, where an estimate equivalent to (5) was obtained for the one-dimensional case. We are thankful to M. L. Lapidus who informed us about $[\mathrm{Fu}]$.

In fact, for $d=1$ a much more detailed analysis is possible. This was done in $[\mathrm{SVe}]$, where it was shown that depending on arithmetic properties of parameters defining $\mu, t^{\delta} n(t)$ behaves at $t=0$ either as a constant or as a function, periodic in $\log t$. Such a detailed result was obtained in $[\mathrm{SVe}]$ on the base of the "renewal equation", well known in Probability and Dynamical Systems; e.g., see [Fe], [Wo] and [LeVa]. Here we also use the 
renewal equation when analyzing an auxiliary combinatorial problem. For this problem we get detailed results of the same type as was obtained in [SVe] for $n(t)$. The eigenvalue distribution function we are dealing with, can be estimated from above and from below through the solution of this combinatorial problem. As a result, for $d \geq 2$ we were able to get only the two-sided estimate (5).

Earlier the renewal equation was applied to various spectral problems involving self-similar sets by Kigami and Lapidus [KLa], Lapidus [La], and Levitin and Vassiliev [LeVa]. For the usage of the renewal theory in other problems related to fractals see [LauW], [St2].

The main purpose of this paper is a detailed exposition of the results announced in [NS]. We give also an analysis of the spectral problem (1) corresponding to the Neumann-type boundary condition. Besides, here we consider a much wider class of domains $X$ than in [NS]; in particular, we treat the case $X=\mathbb{R}^{d}$.

\section{Self-similar measures}

The notions of self-similar set and self-similar measure were introduced by Hutchinson $[\mathrm{Hu}]$. Let $\mathcal{S}=\left\{S_{1}, \ldots, S_{m}\right\}$ be a set of contractive similitudes on $\mathbb{R}^{d}, h_{1}, \ldots, h_{m}$ their coefficients of contraction. Also, let a system of positive numbers ("weights") $\mathbf{p}=\left\{p_{1}, \ldots, p_{m}\right\}$ be given, such that $p_{1}+\ldots+$ $p_{m}=1$. Then there exists a unique nonempty compact set $\mathcal{C}=\mathcal{C}(\mathcal{S}) \subset \mathbb{R}^{d}$ such that $\mathcal{C}=\bigcup_{k=1}^{m} S_{k} \mathcal{C}$, and a unique boundedly supported probability Borelian measure $\mu=\mu(\mathcal{S}, \mathbf{p})$ which satisfies the self-similarity property

$$
\mu=\sum_{k=1}^{m} p_{k} \mu \circ S_{k}^{-1}
$$

Furthermore, $\mathcal{C}(\mathcal{S})=\operatorname{Supp} \mu(\mathcal{S}, \mathbf{p})$. Measures $\mu(\mathcal{S}, \mathbf{p})$, corresponding to the same set $\mathcal{S}$ but to different families $\mathbf{p}$, are mutually singular.

It is $\mu(\mathcal{S}, \mathbf{p})$ that we are interested in. For the rest of the paper $\mu=$ $\mu(\mathcal{S}, \mathbf{p})$.

We always suppose that $\mathcal{S}$ meets the "open set condition" (see $[\mathrm{Hu}]$ ). This means that there exists a bounded open set $\Omega \subset \mathbb{R}^{d}$, such that

$$
\text { 1. } \bigcup_{k=1}^{m} S_{k}(\Omega) \subset \Omega, \quad \text { 2. } S_{k}(\Omega) \bigcap S_{l}(\Omega)=\emptyset \text { for } k \neq l \text {. }
$$


Evidently, (7) implies that $\mathcal{C}(\mathcal{S}) \subseteq \bar{\Omega}$. It follows also that $\sum_{k=1}^{m} h_{k}^{d} \leq 1$. In fact, we will assume that $\Omega$ is a domain (a connected open set). Another assumption is

$$
\mu(\partial \Omega)=0
$$

It is known that otherwise $\mu(\Omega)=0$ (see [LauW]). Assumption (8) is of a rather technical character and can sometimes be withdrawn.

The simplest particular case of a self-similar measure is $\delta_{a}$ (the deltameasure supported at a point $a$ ). We obtain it taking $m=1$.

\section{Embedding theorems}

Let $X \subseteq \mathbb{R}^{d}$ be an arbitrary domain. Since the open set condition (7) does not imply any regularity restriction on the open set, the problem (1) is intimately connected not with the usual Sobolev space $H^{1}$ but with its less familiar counterpart, $L_{2}^{1}$. We recall the definitions of function classes $\stackrel{\circ}{L}_{2}^{1}(X)$ and $L_{2}^{1}(X)$ (all the information needed can be found in $[\mathrm{M}]$ ).

$\stackrel{\circ}{L}_{2}^{1}(X)$ is defined as the closure of $C_{0}^{\infty}(X)$ in the metric of Dirichlet integral $\int_{X}|\nabla u|^{2} d x$. If $d \geq 3$, or if $d=2$ and $\mathbb{R}^{2} \backslash X$ is not a set of zero inner $(2,1)$-capacity, or if $d=1$ and $X \neq \mathbb{R}^{1}$, this is actually a space of functions $([\mathrm{M}], \S 11.2) . \stackrel{\circ}{L}_{2}^{1}(X)$ coincides with the Sobolev space $H_{0}^{1}(X)$ if, for example, $X$ is bounded.

The definition of $L_{2}^{1}(X)$ is as follows:

$$
L_{2}^{1}(X)=\left\{u \in H_{l o c}^{1}(X): \int_{X}|\nabla u|^{2} d x<\infty\right\}
$$

If $u \in L_{2}^{1}(X)$ implies $u \in L_{2}(X)$, then clearly $L_{2}^{1}(X)$ coincides with $H^{1}(X)$ algebraically and topologically. In particular, this is true if $X$ is a bounded domain with the Lipschitz boundary.

$L_{2}^{1}(X)$ can be considered as a Hilbert space with respect to any metric form

$$
\int_{X}|\nabla u|^{2} d x+\left|\int_{X} \psi u d x\right|^{2},
$$

where $\psi \in C_{0}^{\infty}(X)$ is such that $\int_{X} \psi d x \neq 0$. The choice of $\psi$ is arbitrary, and the corresponding norms are equivalent. 
The space

$$
L_{2}(\mu):=L_{2}\left(\mathbb{R}^{d}, \mu\right)
$$

can be identified in a standard way with $L_{2}(\mathcal{C}(S), \mu)$. Let $X \subseteq \mathbb{R}^{d}$ be a domain such that $\Omega \subseteq X$. Our goal here is to study the embeddings of $\stackrel{\circ}{L}_{2}^{1}(X)$ and $L_{2}^{1}(X)$ into $L_{2}(\mu)$. This problem does not arise in the case $d=1$, because then $L_{2}^{1}(X) \subset C(\bar{X})$ and hence the embedding $L_{2}^{1}(X) \subset L_{2}(\mu)$ is bounded and compact for any finite compactly supported $\mu$.

If $d \geq 2$, the problem is more complex. We start with the case $X=\mathbb{R}^{d}$. Denote

$$
A_{k}=h_{k}^{2-d} p_{k}, \quad k=1, \ldots, m, \quad \bar{A}(\mathcal{S}, \mathbf{p})=\max _{k} A_{k} .
$$

Proposition 1. Suppose that (7) (the open set condition) is satisfied. Then

$$
L_{2}^{1}\left(\mathbb{R}^{d}\right) \subset L_{2}(\mu) \quad \text { compactly }
$$

if and only if

$$
\bar{A}(\mathcal{S}, \mathbf{p})<1
$$

Proof. If $d=1$, then (12) is satisfied automatically, and, as was noted above, (11) also holds. So below we discuss only the case $d \geq 2$.

Necessity. Assume that (11) is valid. Then first of all $\mu$ can not have a nonzero delta-component. Indeed, for $d \geq 2$ the Sobolev space $H^{1}$ contains unbounded functions, so the above embedding is not even bounded.

Now suppose that $h_{1}^{2-d} p_{1} \geq 1$. Let $a \in \operatorname{Supp} \mu$. We may assume that $a \neq S_{1}(a)$. Indeed, $m$ contractions $S_{k}$ have finite number (not greater than $m$ ) of fixed points, while Supp $\mu$ must be infinite; otherwise $\mu$ would be a linear combination of delta-measures, which is impossible.

Fix a function $U_{0} \in C_{0}^{\infty}\left(\mathbb{R}^{d}\right)$ such that $U_{0}(a)>0$. Define

$$
U_{n}(x)=p_{1}^{-\frac{n}{2}} U_{0}\left(S_{1}^{-n}(x)\right),
$$

then, in view of the self-similarity of $\mu, \int\left|U_{n}\right|^{2} d \mu=\int\left|U_{0}\right|^{2} d \mu$. Clearly, $U_{n} \in H^{1}\left(\mathbb{R}^{d}\right) \subset L_{2}^{1}\left(\mathbb{R}^{d}\right)$. Moreover, $\left\{U_{n}\right\}$ is bounded in $L_{2}^{1}\left(\mathbb{R}^{d}\right)$ :

$$
\begin{aligned}
& \left\|U_{n}\right\|_{L_{2}^{1}\left(\mathbb{R}^{d}\right)}^{2} \\
& =\int_{\mathbb{R}^{d}}\left|\nabla U_{n}\right|^{2} d x+\left|\int_{\mathbb{R}^{d}} \psi U_{n} d x\right|^{2} \leq \int_{\mathbb{R}^{d}}\left|\nabla U_{n}\right|^{2} d x+C \int_{\mathbb{R}^{d}}\left|U_{n}\right|^{2} d x \\
& =\left(p_{1} h_{1}^{2-d}\right)^{-n}\left(\int_{\mathbb{R}^{d}}\left|\nabla U_{0}\right|^{2} d x+C h_{1}^{2 n} \int_{\mathbb{R}^{d}}\left|U_{0}\right|^{2} d x\right) \leq C^{\prime}\left\|U_{0}\right\|_{H^{1}\left(\mathbb{R}^{d}\right)}^{2} .
\end{aligned}
$$


Now recall that $S_{1}(a) \neq a$. If $b$ is the fixed point of $S_{1}$, then $\left|S_{1}^{n}(a)-b\right|=$ $h_{1}^{n}|a-b|$ for any $n$. Therefore, $U_{0}$ may be chosen so that Supp $U_{n}$ are mutually disjoint. Thus

$$
\int\left|U_{n}-U_{m}\right|^{2} d \mu=\int\left|U_{n}\right|^{2} d \mu+\int\left|U_{m}\right|^{2} d \mu=2 \int\left|U_{0}\right|^{2} d \mu>0
$$

which, together with (14), contradicts the compactness of the embedding.

Sufficiency. Let $h_{k}^{2-d} p_{k}<1, \quad k=1, \ldots, m$. Then for $\varepsilon$ positive and sufficiently small we have $\left(h_{k}^{2-d}\right)^{1+\varepsilon} p_{k}<1$, any $k$. Choose $q$ such that $2<q<2+2 \varepsilon$. We will prove first that under our assumptions the embedding $H^{1}\left(\mathbb{R}^{d}\right) \subset L_{q}(\mu)$ is compact. For a finite measure $\mu$, this yields the compactness of $H^{1}\left(\mathbb{R}^{d}\right) \subset L_{2}(\mu)$. (11) follows from here because $\mu$ is compactly supported.

We will need the following result (see [M], Theorems 3 and 4 in $\S 8.8$ ):

The embedding $H^{1}\left(\mathbb{R}^{d}\right) \subset L_{q}(\mu)$ is compact if and only if

$$
\begin{aligned}
& \lim _{\delta \rightarrow+0} \sup _{x \in \mathbb{R}^{d}, \rho \in(0, \delta)} \rho^{1-\frac{d}{2}}[\mu(B(x, \rho))]^{\frac{1}{q}}=0 \quad \text { for } d>2, \\
& \lim _{\delta \rightarrow+0} \sup _{x \in \mathbb{R}^{d}, \rho \in(0, \delta)}|\log \rho|^{\frac{1}{2}}[\mu(B(x, \rho))]^{\frac{1}{q}}=0 \quad \text { for } d=2,
\end{aligned}
$$

where $B(x, \rho)$ denotes the ball of radius $\rho$ centered at $x$.

Now we concentrate on the proof of (16) and (17).

For a multiindex $\mathbf{j}=\left(j_{1}, \ldots, j_{n}\right) \in\{1, \ldots, m\}^{n}$ we denote

$$
\begin{aligned}
S(\mathbf{j}) & =S_{j_{n}} \ldots S_{j_{1}}, \\
\Omega(\mathbf{j}) & =S(\mathbf{j}) \Omega, \\
p(\mathbf{j}) & =p_{j_{1}} \ldots p_{j_{n}}, \\
h(\mathbf{j}) & =h_{j_{1}} \ldots h_{j_{n}}, \\
A(\mathbf{j}) & =A_{j_{1}} \ldots A_{j_{n}} .
\end{aligned}
$$

Also, denote

$$
p_{\max }=\max _{k=1, \ldots, m} p_{k}, \quad h_{\min }=\min _{k=1, \ldots, m} h_{k} .
$$


Here we apply the following result (see [Fa], Lemma 8.5 and the proof of Theorem 8.6):

Let the open set condition (7) be satisfied, and $0<\rho<1$. Then there exist constants $c, l$ and a family of multiindices $\mathbf{j}_{1}, \ldots, \mathbf{j}_{r}$ satisfying

$$
\Omega\left(\mathbf{j}_{\alpha}\right) \cap \Omega\left(\mathbf{j}_{\beta}\right)=\emptyset \quad \text { for } \alpha \neq \beta
$$

and

$$
c \rho \leq h\left(\mathbf{j}_{\alpha}\right) \leq \rho, \quad \text { any } \alpha,
$$

such that any ball $B_{\rho}$ of radius $\rho$ intersects no more than $l$ of the sets $\Omega\left(\mathbf{j}_{\alpha}\right)$.

Therefore, for $d>2$ we get:

$$
\begin{aligned}
& \left.\rho^{1-\frac{d}{2}}[\mu(B(x, \rho))]^{\frac{1}{q}} \leq \rho^{1-\frac{d}{2}}\left[l \max _{\alpha} \mu\left(\overline{\Omega\left(\mathbf{j}_{\alpha}\right.}\right)\right)\right]^{\frac{1}{q}} \\
& \quad \leq l^{\frac{1}{q}} \rho^{1-\frac{d}{2}}\left[\max _{h\left(\mathbf{j}_{\alpha}\right) \leq \rho} p\left(\mathbf{j}_{\alpha}\right)\right]^{\frac{1}{q}} \leq l^{\frac{1}{q}} \rho^{1-\frac{d}{2}} \rho^{(d-2)(1+\varepsilon) \frac{1}{q}}=l^{\frac{1}{q}} \rho^{(d-2)\left(\frac{1+\varepsilon}{q}-\frac{1}{2}\right) .}
\end{aligned}
$$

Since $q<2(1+\varepsilon)$, we have $\frac{1+\varepsilon}{q}-\frac{1}{2}>0$. Hence

$$
\lim _{\delta \rightarrow+0} \sup _{x \in \mathbb{R}^{d}, \rho \in(0, \delta)} \rho^{1-\frac{d}{2}}[\mu(B(x, \rho))]^{\frac{1}{q}} \leq l^{\frac{1}{q}} \lim _{\delta \rightarrow+0} \delta^{(d-2)\left(\frac{1+\varepsilon}{q}-\frac{1}{2}\right)}=0 .
$$

For $d=2$, we get in a similar way:

$$
|\log \rho|^{\frac{1}{2}}[\mu(B(x, \rho))]^{\frac{1}{q}} \leq l^{\frac{1}{q}}|\log \rho|^{\frac{1}{2}}\left[\max _{h\left(\mathbf{j}_{\alpha}\right) \leq \rho} p\left(\mathbf{j}_{\alpha}\right)\right]^{\frac{1}{q}} .
$$

$h\left(\mathbf{j}_{\alpha}\right) \leq \rho$ implies $\left(h_{\text {min }}\right)^{\left|\mathbf{j}_{\alpha}\right|} \leq h\left(\mathbf{j}_{\alpha}\right) \leq \rho$, so $\left|\mathbf{j}_{\alpha}\right| \geq\left(\log h_{m i n}\right)^{-1} \log \rho$. Hence

$$
\max _{h\left(\mathbf{j}_{\alpha}\right) \leq \rho} p\left(\mathbf{j}_{\alpha}\right) \leq\left(p_{\max }\right)^{\left|\mathbf{j}_{\alpha}\right|} \leq \exp \left(\frac{\log p_{\max }}{\log h_{\min }} \log \rho\right)=\rho^{\frac{\log p_{\max }}{\log h_{\min }}},
$$

and we obtain

$$
\lim _{\delta \rightarrow+0} \sup _{x \in \mathbb{R}^{d}, \rho \in(0, \delta)}|\log \rho|^{\frac{1}{2}}[\mu(B(x, \rho))]^{\frac{1}{q}} \leq l^{\frac{1}{q}} \lim _{\delta \rightarrow+0}|\log \delta|^{\frac{1}{2}} \delta^{\frac{\log p_{\max }}{q \log h_{\min }}}=0 .
$$

So we have proved that for $d \geq 2$ the embedding $H^{1}\left(\mathbb{R}^{d}\right) \subset L_{q}(\mu)$ is compact for some $q>2$, which implies (11).

We could not obtain a sufficient condition for the embedding $L_{2}^{1}\left(\mathbb{R}^{d}\right) \subset$ $L_{2}(\mu)$ to be bounded. Clearly, $\bar{A}(\mathcal{S}, \mathbf{p}) \leq 1$ is necessary, but it is not sufficient even if $d=2$. Indeed, if $p_{1} h_{1}^{d-2}=p_{1}=1$, then we have only one contraction $S_{1}$, and $\mu$ is a delta-measure. So if $d=2$, the boundedness and the compactness of the embedding are equivalent. It is unknown to us whether this is still true for $d>2$. 
Proposition 2. Suppose that (7) is satisfied and $d \geq 2$. Let $X \subseteq \mathbb{R}^{d}$ be a domain such that $\Omega \subseteq X$. If $d=2$, suppose also that $\mathbb{R}^{2} \backslash X$ has nonzero inner (2,1)-capacity. If (12) is satisfied, that is, $\bar{A}(\mathcal{S}, \mathbf{p})<1$, then

$$
L_{2}^{\circ}(X) \subset L_{2}(\mu) \quad \text { compactly }
$$

If, in addition, $\mu(\partial \Omega)=0$, then (12) is also necessary for the compactness of $L_{2}^{\circ}(X) \subset L_{2}(\mu)$.

Proof. The proof is easily reduced to the one of Proposition 1.

Necessity. Since $\mu(\partial \Omega)=0$, we can choose $a \in \operatorname{Supp} \mu$ so that $a \in \Omega$. Moreover, Supp $\mu \backslash \partial \Omega$ is infinite (otherwise $\mu$ includes a delta-measure). Therefore $a$ again can be taken in such a way that $a \neq S_{1}(a)$.

Suppose that $h_{1}^{2-d} p_{1} \geq 1$. Define $U_{n}$ as in (13). Clearly, we may assume Supp $U_{n} \subset \Omega$. So if $u_{n}:=\left.U_{n}\right|_{X}$, then $u_{n} \in \stackrel{\circ}{L}_{2}^{1}(X)$ and

$$
\left\|u_{n}\right\|_{L_{2}^{1}(X)}^{2}=\int_{\mathbb{R}^{d}}\left|\nabla U_{n}\right|^{2} d x \leq\left\|U_{0}\right\|_{H^{1}\left(\mathbb{R}^{d}\right)}^{2} .
$$

Therefore, $\left\{u_{n}\right\}$ is bounded in $\stackrel{\circ}{L}_{2}^{1}(X)$. The remaining part of the proof is the same.

Sufficiency. Follows evidently from Proposition 1.

Before going over to $L_{2}^{1}(X)$, let us discuss in more detail the sense of the embedding considered. Suppose first that $\mu(\partial \Omega)=0$. Then the integral

$$
Q_{\mu}[u]=\int|u|^{2} d \mu
$$

is well defined on the set $L_{2}^{1}(X) \cap C^{\infty}(X)$, which is dense in $L_{2}^{1}(X)$. If $Q_{\mu}$ is bounded in $L_{2}^{1}(X)$, that is, if for any $u \in L_{2}^{1}(X) \cap C^{\infty}(X)$ we have

$$
Q_{\mu}[u] \leq C\left(\int_{X}|\nabla u|^{2}+\left|\int_{X} \psi u d x\right|^{2}\right)
$$

then we extend $Q_{\mu}$ to the whole of $L_{2}^{1}(X)$ by continuity. For $L_{2}^{1}\left(\mathbb{R}^{d}\right)$ and $\stackrel{\circ}{L}_{2}^{1}(X)$ the sense of the embeddings considered is the same, with $C_{0}^{\infty}\left(\mathbb{R}^{d}\right)$ or $C_{0}^{\infty}(X)$ as the initial domain of $Q_{\mu}$. 
The situation changes if $\mu(\partial \Omega)=1$, because then we can not start with $u \in C^{\infty}(X)$. In order that $Q_{\mu}[u]$ be well defined on $L_{2}^{1}(X)$, we need an additional assumption:

$$
L_{2}^{1}(X) \cap C^{\infty}(X \cup \operatorname{Supp} \mu) \text { is dense in } L_{2}^{1}(X) .
$$

Now the same scheme as above works; if (21) is valid for any $u \in L_{2}^{1}(X) \cap$ $C^{\infty}(X \cup \operatorname{Supp} \mu)$, then we extend $Q_{\mu}$ to $L_{2}^{1}(X)$ by continuity.

The condition (22) withdraws some pathological cases. Say, if $d=2, X$ is the unit disc with a cut along a radius and $\mu$ is supported on this radius, then $Q_{\mu}[u]$ makes no sense on $L_{2}^{1}(X)$.

Another notion we need below is the extension property for $L_{2}^{1}$.

We say that a domain $X \subset \mathbb{R}^{d}$ admits the extension property for $L_{2}^{1}$ and write

$$
X \in \mathcal{E}\left(L_{2}^{1}\right)
$$

if there exists a continuous linear operator $\Pi: L_{2}^{1}(X) \rightarrow L_{2}^{1}\left(\mathbb{R}^{d}\right)$ such that $\left.\Pi f\right|_{X}=f$ for any $f \in L_{2}^{1}(X)$. The extension property for $H^{1}$ and the class $\mathcal{E}\left(H^{1}\right)$ are defined in the same way.

The following statement (due to V. Maz'ya) shows that for a bounded $X$ these two properties are equivalent. (If $X$ is unbounded, then this is not the case.)

Lemma 1. Let $X \subset \mathbb{R}^{d}$ be a bounded domain. Then

$$
X \in \mathcal{E}\left(L_{2}^{1}\right) \Longleftrightarrow X \in \mathcal{E}\left(H^{1}\right) .
$$

Proof. The implication " $\Longrightarrow$ " is evident. Conversely, if $X \in \mathcal{E}\left(H^{1}\right)$, then $H^{1}(X) \subset L_{q}(X)$ for some $q>2$. So for any $u \in H^{1}(X)$

$$
\left(\int_{X}|u|^{q} d x\right)^{\frac{2}{q}} \leq C\left(\int_{X}|\nabla u|^{2} d x+\int_{X_{0}}|u|^{2} d x+\int_{X^{\prime}}|u|^{2} d x\right),
$$

where $X_{0} \subset X$ is a domain with the Lipschitz boundary, and $X^{\prime}=X \backslash X_{0}$. Estimating the last term on the right-hand side of (23) by the Hölder inequality, we get

$$
\begin{aligned}
& \left(\int_{X}|u|^{q} d x\right)^{\frac{2}{q}} \leq \\
& \quad C\left(\int_{X}|\nabla u|^{2} d x+\int_{X_{0}}|u|^{2} d x\right)+C\left(\operatorname{meas} X^{\prime}\right)^{1-\frac{2}{q}}\left(\int_{X^{\prime}}|u|^{q} d x\right)^{\frac{2}{q}}
\end{aligned}
$$


Choosing $X_{0}$ in such a way that $C\left(\operatorname{meas} X^{\prime}\right)^{1-\frac{2}{q}}<\frac{1}{2}$, we obtain

$$
\|u\|_{L_{2}(X)} \leq C_{1}\|u\|_{L_{q}(X)} \leq C_{2}\left(\int_{X}|\nabla u|^{2} d x+\int_{X_{0}}|u|^{2} d x\right)^{\frac{1}{2}} .
$$

Since the right-hand side of (25) is an equivalent norm in $L_{2}^{1}(X)$ and $H^{1}(X)$ is dense in $L_{2}^{1}(X)$, we are done.

It follows from this Lemma that for bounded $X \in \mathcal{E}\left(H^{1}\right)$ we have $L_{2}^{1}(X)=H^{1}(X)$. Recall that any bounded domain with the Lipschitz boundary belongs to $\mathcal{E}\left(H^{1}\right)$; e.g., see [Ste]. For the rest of the paper we denote $\mathcal{E}=\mathcal{E}\left(L_{2}^{1}\right)$.

Notice that if $X \in \mathcal{E}$, then (22) is automatically satisfied, because in this case $L_{2}^{1}(X) \cap C^{\infty}(\bar{X})$ is dense in $L_{2}^{1}(X)$.

Proposition 3. Suppose that (7) is satisfied. Let $d \geq 2$ and $X \subseteq \mathbb{R}^{d}$ be a domain such that $\Omega \subseteq X$. If $\mu(\partial \Omega)=1$, then let also (22) be satisfied. If

$$
L_{2}^{1}(X) \subset L_{2}(\mu) \quad \text { compactly, }
$$

then (12) is satisfied, that is, $\bar{A}(\mathcal{S}, \mathbf{p})<1$.

If, in addition, there exists a domain $Y \in \mathcal{E}$ such that $\Omega \subseteq Y \subseteq X$, then (12) is also sufficient for compactness of $L_{2}^{1}(X) \subset L_{2}(\mu)$.

Proof. The proof is quite similar to the one of Proposition 1; the slight changes needed are clear.

\section{The operators $T_{\mu, X}^{D}$ and $T_{\mu, X}^{N}$. Formulation of the main results}

Here we give an accurate statement of the problems to be considered below.

If the conditions of the sufficiency part of Proposition 2 are satisfied, then $Q_{\mu}[u]$ generates in $\stackrel{\circ}{L}_{2}^{1}(X)$ a compact, self-adjoint and nonnegative operator, which we denote by $T_{\mu, X}^{D}$. The equality

$$
T_{\mu, X}^{D} u=f, \quad u, f \in \stackrel{\circ}{L}_{2}^{1}(X)
$$

means that

$$
\int_{X} \nabla f \cdot \nabla \bar{v} d x=\int_{X} u \bar{v} d \mu, \quad \text { any } v \in \stackrel{\circ}{L}_{2}^{1}(X) .
$$


In particular, every eigenpair $(\lambda, u)$ meets the equation (1), with $\mathcal{V}=$ $\stackrel{\circ}{L}_{2}^{1}(X)$.

To define $T_{\mu, X}^{N}$, we need to introduce a special metric form on $L_{2}^{1}(X)$.

Assume that we are in the conditions of the sufficiency part of Proposition 3, so that the embedding $L_{2}^{1}(X) \subset L_{2}(\mu)$ is compact. Along with the quadratic functional $Q_{\mu}[u]$ introduce a linear functional

$$
\phi_{\mu}(u)=\int u d \mu .
$$

This makes sense for $u \in C^{\infty}(X)$ if $\mu(\partial \Omega)=0$, or for $u \in C^{\infty}(X \cup \operatorname{Supp} \mu)$ if $\mu(\partial \Omega)=1$. Clearly,

$$
\left|\phi_{\mu}(u)\right|^{2} \leq Q_{\mu}[u]
$$

so $\phi_{\mu}$ is bounded on $L_{2}^{1}(X)$. As usual, we extend $\phi_{\mu}$ to the whole of $L_{2}^{1}(X)$ by continuity. Notice that according to our definitions, we have for any $u \in L_{2}^{1}(X)$

$$
Q_{\mu}[u]=Q_{\mu}\left[\left.u\right|_{\Omega}\right] \quad \text { and } \quad \phi_{\mu}(u)=\phi_{\mu}\left(\left.u\right|_{\Omega}\right) .
$$

Consider now the metric form on $L_{2}^{1}(X)$ :

$$
D_{\mu}^{2}[u]=\int_{X}|\nabla u|^{2} d x+\left|\phi_{\mu}(u)\right|^{2} .
$$

Lemma 2. Suppose that the assumptions of Proposition 3 are satisfied. Then $L_{2}^{1}(X)$ is complete with respect to $D_{\mu}$.

Proof. Our arguments are quite standard. Let $\left\{u_{k}\right\}$ be a Cauchy sequence in the metric $D_{\mu}$, then $\left\{\left.u_{k}\right|_{Y}\right\}$ is a Cauchy sequence in $H^{1}(Y)$. So there exists $v \in H^{1}(Y)$ such that $\left\|\left.u_{k}\right|_{Y}-v\right\|_{H^{1}(Y)} \rightarrow 0$. Besides, it follows from the completeness of $L_{2}^{1}(X)$ factorized by constants $([\mathrm{M}], \S 1.1 .13)$, that there exists a function $u \in L_{2}^{1}(X)$ such that $\int_{X}\left|\nabla\left(u_{k}-u\right)\right|^{2} d x \rightarrow 0$, and therefore $\int_{Y}\left|\nabla\left(u_{k}-u\right)\right|^{2} d x \rightarrow 0$. It follows that $u-v=C=$ const on $Y$. Now, $u^{\prime}:=u-C \in L_{2}^{1}(X)$ and $u_{k} \rightarrow u^{\prime}$ in the metric $D_{\mu}$.

Suppose now that the conditions of the sufficiency part of Proposition 3 are satisfied. Then by $T_{\mu, X}^{N}$ we denote the compact, self-adjoint and nonnegative operator generated by $Q_{\mu}[u]$ in $L_{2}^{1}(X)$ with $D_{\mu}$ as the metric form. The equality

$$
T_{\mu, X}^{N} u=\lambda u, \quad u \in L_{2}^{1}(X)
$$


means that

$$
\lambda\left(\int_{X} \nabla u \cdot \nabla \bar{v} d x+\int_{\bar{X}} u d \mu \cdot \int_{\bar{X}} \bar{v} d \mu\right)=\int_{\bar{X}} u \bar{v} d \mu, \quad \text { any } v \in L_{2}^{1}(X) .
$$

The equation (29) has the trivial eigenvalue $\lambda=1$, with the corresponding eigenfunction $u_{0} \equiv 1$. For any eigenfunction orthogonal to $u_{0},(29)$ is equivalent to (1), with $\mathcal{V}=\left\{v \in L_{2}^{1}(X): \int_{\bar{X}} v d \mu=0\right\}$.

Our interest lies in the behavior of the eigenvalues $\lambda_{k}\left(T_{\mu, X}^{D}\right)$ or $\lambda_{k}\left(T_{\mu, X}^{N}\right)$; we shorten the notation of both operators to $T_{\mu}$ when it is unambiguous. As usual, we express this behaviour in terms of the corresponding distribution function

$$
n\left(t, T_{\mu}\right)=\#\left\{k: \lambda_{k}\left(T_{\mu}\right)>t\right\} .
$$

Theorem 1. Let (7) and (12) be satisfied, and assume $\Omega \in \mathcal{E}$. Let $X$ be a domain in $\mathbb{R}^{d}, d \geq 2$, such that $\Omega \subseteq X$. If $d=2$, then let $\mathbb{R}^{2} \backslash X$ have nonzero inner (2,1)-capacity. Define $\delta>0$ as the unique solution of

$$
\sum_{k=1}^{m} A_{k}^{\delta}=\sum_{k=1}^{m}\left(h_{k}^{2-d} p_{k}\right)^{\delta}=1 .
$$

Then there exists a constant $C>0$ such that for $T_{\mu}=T_{\mu, X}^{D}$

$$
n\left(t, T_{\mu}\right) \leq C t^{-\delta}, \quad \text { for any } t>0 .
$$

If, in addition, $\mu(\partial \Omega)=0$, then there exist positive constants $c$ and $t_{0}$ such that for $T_{\mu}=T_{\mu, X}^{D}$

$$
n\left(t, T_{\mu}\right) \geq c t^{-\delta}, \text { for any } t \in\left(0, t_{0}\right) .
$$

Theorem 2. Let (7) and (12) be satisfied, and assume $\Omega \in \mathcal{E}$. Let $X$ be a domain in $\mathbb{R}^{d}, d \geq 2$, such that $\Omega \subseteq X$. If $\mu(\partial \Omega)=1$, then let also (22) be satisfied. Define $\delta>0$ as the unique solution of (30).

Then the results of Theorem 1 are valid for $T_{\mu}=T_{\mu, X}^{N}$.

\section{Proof of the main results: basic construction}

Theorems 1 and 2 will be proved simultaneously. We use the variational approach. Self-similarity of $\mu$ allows us to present a construction giving for $n\left(t, T_{\mu}\right)$ estimates of order $O\left(t^{-\delta}\right)$ both from above and from below. 
Let a finite set of multiindices $\mathbf{j}_{1}, \ldots, \mathbf{j}_{r}$, whose entries are $1, \ldots, m$ and whose length is not fixed, be chosen in such a way that the family $\Xi=$ $\left\{\Omega\left(\mathbf{j}_{1}\right), \ldots, \Omega\left(\mathbf{j}_{r}\right)\right\}$ satisfies

$$
\text { 1. } \Omega\left(\mathbf{j}_{\alpha}\right) \cap \Omega\left(\mathbf{j}_{\beta}\right)=\emptyset \text { for } \alpha \neq \beta, \quad \text { 2. } \mu\left(\cup_{\alpha=1}^{r} \Omega\left(\mathbf{j}_{\alpha}\right)\right)=1 .
$$

It was supposed that $\Omega \in \mathcal{E}$ in both Theorems 1 and 2 . So the conditions of Proposition 3 are satisfied for $X=\Omega$. This yields

$$
\int_{\bar{\Omega}}|u|^{2} d \mu \leq K\left(\int_{\Omega}|\nabla u|^{2} d x+\left|\int_{\bar{\Omega}} u d \mu\right|^{2}\right), \quad \text { any } u \in L_{2}^{1}(\Omega),
$$

with $K=\left\|T_{\mu, \Omega}^{N}\right\|^{2}$.

Let $\mathcal{V}=\stackrel{\circ}{L}_{2}^{1}(X)$ or $\mathcal{V}=\left\{v \in L_{2}^{1}(X): \int_{\bar{X}} v d \mu=0\right\}$, for Theorems 1 or 2 respectively. Consider the subspace $F \subset \mathcal{V}$ :

$$
F=\left\{u \in \mathcal{V}: \int_{\bar{\Omega}\left(\mathbf{j}_{\alpha}\right)} u d \mu=0, \quad \alpha=1, \ldots, r\right\}
$$

Clearly, $\operatorname{codim} F \leq r$. For any $u \in F$ and any $\alpha=1, \ldots, r$ (33) yields:

$$
\int_{\bar{\Omega}}\left|u\left(S^{-1}\left(\mathbf{j}_{\alpha}\right) x\right)\right|^{2} d \mu \leq K \int_{\Omega}\left|\nabla u\left(S^{-1}\left(\mathbf{j}_{\alpha}\right) x\right)\right|^{2} d x
$$

so

$$
\begin{aligned}
& \int_{\overline{\Omega\left(\mathbf{j}_{p}\right)}}|u(x)|^{2} d \mu=p\left(\mathbf{j}_{\alpha}\right) \int_{\bar{\Omega}}\left|u\left(S^{-1}\left(\mathbf{j}_{\alpha}\right) x\right)\right|^{2} d \mu \\
& \leq K p\left(\mathbf{j}_{\alpha}\right) h\left(\mathbf{j}_{\alpha}\right)^{2-d} \int_{\Omega\left(\mathbf{j}_{\alpha}\right)}|\nabla u(x)|^{2} d x=K A\left(\mathbf{j}_{\alpha}\right) \int_{\Omega\left(\mathbf{j}_{\alpha}\right)}|\nabla u(x)|^{2} d x,
\end{aligned}
$$

with the same constant factor $K$ as in (33).

Denote

$$
\begin{aligned}
& t_{+}=t_{+}(\Xi)=\max \left\{A\left(\mathbf{j}_{\alpha}\right), \alpha=1, \ldots, r\right\}, \\
& t_{-}=t_{-}(\Xi)=\min \left\{A\left(\mathbf{j}_{\alpha}\right), \alpha=1, \ldots, r\right\} .
\end{aligned}
$$

Then for any $u \in F$ we get:

$$
\begin{aligned}
\int_{\bar{\Omega}}|u|^{2} d \mu= & \sum_{\alpha=1}^{r} \int_{\overline{\Omega\left(\mathbf{j}_{\alpha}\right)}}|u(x)|^{2} d \mu \leq K \sum_{\alpha=1}^{r} A\left(\mathbf{j}_{\alpha}\right) \int_{\Omega\left(\mathbf{j}_{\alpha}\right)}|\nabla u(x)|^{2} d x \\
& \leq K t_{+} \sum_{\alpha=1}^{r} \int_{\Omega\left(\mathbf{j}_{\alpha}\right)}|\nabla u(x)|^{2} d x \leq K t_{+} \int_{X}|\nabla u(x)|^{2} d x .
\end{aligned}
$$


So we have constructed a subspace of codimension not greater than $r$ on which

$$
\int_{\bar{\Omega}}|u|^{2} d \mu \leq K t_{+} \int_{X}|\nabla u(x)|^{2} d x
$$

Therefore, in view of the variational principle, we obtain both for $T_{\mu, X}^{D}$ and $T_{\mu, X}^{N}$ :

$$
n\left(K t_{+}, T_{\mu}\right) \leq r .
$$

Now, we turn to the estimates from below. In the proof of Theorem 2, we will deal here with $L_{2}^{1}(X)$ rather than with $\mathcal{V}$. Since the codimension of $\mathcal{V}$ in $L_{2}^{1}(X)$ is 1 , this suffices for the proof of $(32)$.

Recall that here we suppose $\mu(\partial \Omega)=0$. Let $a \in \operatorname{Supp} \mu$. We may assume $a \in \Omega$. Fix a nonnegative function $u_{0} \in C_{0}^{\infty}(\Omega)$ such that $u_{0}(a)>0$. Define

$$
u_{\mathbf{j}_{\alpha}}(x)= \begin{cases}u_{0}\left(S^{-1}\left(\mathbf{j}_{\alpha}\right) x\right), & x \in \Omega\left(\mathbf{j}_{\alpha}\right) \\ 0, & x \in X \backslash \Omega\left(\mathbf{j}_{\alpha}\right),\end{cases}
$$

then $u_{\mathbf{j}_{\alpha}} \in \stackrel{\circ}{L_{2}^{1}}(X)$. Moreover, Supp $u_{\mathbf{j}_{\alpha}} \subset \Omega\left(\mathbf{j}_{\alpha}\right)$, so the supports of $u_{\mathbf{j}_{\alpha}}$ for different $\alpha$ are mutually disjoint. Hence $u_{\mathbf{j}_{\alpha}}$ are mutually orthogonal both in $L_{2}^{1}$ and in $L_{2}(\mu)$ scalar products. We have

$$
\int_{X}\left|u_{\mathbf{j}_{\alpha}}\right|^{2} d \mu=\int_{\Omega\left(\mathbf{j}_{\alpha}\right)}\left|u_{\mathbf{j}_{\alpha}}\right|^{2} d \mu=p\left(\mathbf{j}_{\alpha}\right) \int_{\Omega}\left|u_{0}\right|^{2} d \mu
$$

and

$$
\begin{array}{r}
\int_{X}\left|\nabla u_{\mathbf{j}_{\alpha}}\right|^{2} d x+\left|\int u_{\mathbf{j}_{\alpha}} d \mu\right|^{2}=\int_{\Omega\left(\mathbf{j}_{\alpha}\right)}\left|\nabla u_{\mathbf{j}_{\alpha}}\right|^{2} d x+\left|\int_{\Omega\left(\mathbf{j}_{\alpha}\right)} u_{\mathbf{j}_{\alpha}} d \mu\right|^{2} \\
=\left(h\left(\mathbf{j}_{\alpha}\right)\right)^{d-2} \int_{\Omega}\left|\nabla u_{0}\right|^{2} d x+p\left(\mathbf{j}_{\alpha}\right)\left|\int_{\Omega} u_{0} d \mu\right|^{2} \\
\leq\left(h\left(\mathbf{j}_{\alpha}\right)\right)^{d-2}\left(\int_{\Omega}\left|\nabla u_{0}\right|^{2} d x+\left|\int_{\Omega} u_{0} d \mu\right|^{2}\right) .
\end{array}
$$

So we have constructed a subspace of dimension $r$ on which

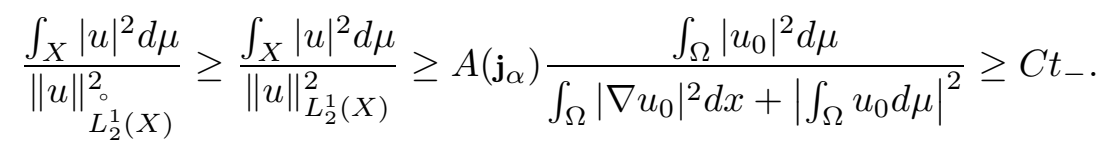


Applying again the variational principle, we get both for $T_{\mu, X}^{D}$ and $T_{\mu, X}^{N}$ :

$$
n\left(C t_{-}, T_{\mu}\right) \geq r
$$

Now we describe a suitable sequence of families

$$
\Xi_{N}=\left\{\Omega\left(\mathbf{j}_{1}^{N}\right), \ldots, \Omega\left(\mathbf{j}_{r(N)}^{N}\right)\right\}
$$

Take $\Xi_{0}=\{\Omega\}$. Suppose $\Xi_{N}$ is already constructed. Let $\mathbf{j}_{0}$ be a multiindex for which $\Omega\left(\mathbf{j}_{0}\right) \in \Xi_{N}$ and

$$
A\left(\mathbf{j}_{0}\right)=t_{+}\left(\Xi_{N}\right)
$$

Then $\Xi_{N+1}$ is obtained from $\Xi_{N}$ by the replacement of $\Omega\left(\mathbf{j}_{0}\right)$ by the collection $S_{1} \Omega\left(\mathbf{j}_{0}\right), \ldots, S_{m} \Omega\left(\mathbf{j}_{0}\right)$. (If there are several such multiindices $\mathbf{j}_{0}$, we do the same with all the corresponding sets $\Omega\left(\mathbf{j}_{0}\right)$.)

It is easy to see that for the sequence $\left\{\Xi_{N}\right\}_{N=0}^{\infty}$ we have

$$
t_{+}\left(\Xi_{N}\right) \leq \bar{A}(\mathcal{S}, \mathbf{p}) t_{-}\left(\Xi_{N}\right)
$$

Now Theorems 1 and 2 can be proved with the help of an auxiliary combinatorial problem.

\section{Combinatorial problem}

Consider a class of paths on $\left(\mathbb{Z}_{+}\right)^{m}$. The initial point of each path is 0 and every link is parallel to one of the coordinate axes and goes in positive direction. Let a linear function $L(x)$ in $\mathbb{R}^{m}$ be given:

$$
L(x)=L\left(x_{1}, \ldots, x_{m}\right)=c_{1} x_{1}+\ldots+c_{m} x_{m}, \quad c_{k}>0, \quad k=1, \ldots, m .
$$

Denote

$$
c_{\min }=\min _{k=1, \ldots, m} c_{k}, \quad c_{\max }=\max _{k=1, \ldots, m} c_{k} .
$$

Fix $s \geq 0$. Let $\mathcal{N}(s)$ be the family of paths whose last link intersects the hyperplane $L(x)=s$. (The path is in $\mathcal{N}(s)$ if the endpoint of its last link belongs to this hyperplane, and is not in $\mathcal{N}(s)$, if the starting point does.) Denote $\# \mathcal{N}(s)=N(s)$. Our aim is to estimate $N(s)$. This we will do in an "indirect" way. Namely, let $\mathcal{W}(s)$ be the family of paths that lie in 
the pyramid $\left(\mathbb{R}_{+}\right)^{m} \bigcap\{L(x) \leq s\}$ and cannot be extended inside it and let $W(s)=\# \mathcal{W}(s)$. For $s<0$ we set $W(s)=0$. Then

$$
W(s) \leq N(s) \leq W\left(s+c_{\max }\right)
$$

Indeed, consider a path from $\mathcal{W}(s)$. The endpoint of its last link either lies on the hyperplane $L(x)=s$-then this path belongs also to $\mathcal{N}(s)$-or it is beneath the hyperplane; in this case the path may be continued to become the one from $\mathcal{N}(s)$. So to any path of $\mathcal{W}(s)$ corresponds at least one path from $\mathcal{N}(s)$, and this correspondence is clearly injective. Hence $W(s) \leq N(s)$.

A similar argument shows that $N(s) \leq W\left(s+c_{\max }\right)$; we should only take into account that the endpoints $x^{e}=\left(x_{1}^{e}, \ldots, x_{m}^{e}\right)$ of paths from $\mathcal{N}(s)$ satisfy

$$
s \leq L\left(x^{e}\right)=c_{1} x_{1}^{e}+\ldots+c_{m} x_{m}^{e}<s+c_{\max } .
$$

Now notice that $W(s)$ satisfies a simple functional equation:

$$
W(s)=W\left(s-c_{1}\right)+\ldots+W\left(s-c_{m}\right)+\Delta(s),
$$

where $\Delta(s)=1$ for $s<c_{\text {min }}$ and $\Delta(s)=0$ for $s \geq c_{\text {min }}$ (the verification is straightforward). Define $\delta$ as the unique nonnegative solution of the equation

$$
e^{-\delta c_{1}}+\ldots+e^{-\delta c_{m}}=1 .
$$

Denoting $F(s)=e^{-s \delta} W(s)$, we rewrite (44) as

$$
F(s)=e^{-\delta c_{1}} F\left(s-c_{1}\right)+\ldots+e^{-\delta c_{m}} F\left(s-c_{m}\right)+e^{-\delta s} \Delta(s) .
$$

This is a particular case of the so called renewal equation. The solvability results for this equation can be borrowed e.g. from [Fe], Chapter XI.1. We come to the following statement:

Proposition 4. 1) If at least one of the ratios $\frac{c_{k}}{c_{l}}$ is irrational ("nonarithmetic case"), then there exists a positive constant $B$ such that

$$
e^{-\delta s} W(s) \rightarrow B, \quad s \rightarrow \infty .
$$

2) If all the ratios $\frac{c_{k}}{c_{l}}$ are rational ("arithmetic case"), then there exists bounded, positive and bounded away from zero periodic function $\psi(s)$ on $\mathbb{R}^{d}$ such that

$$
e^{-\delta s} W(s)-\psi(s) \rightarrow 0, \quad s \rightarrow \infty
$$


In fact, the complete formulation of this Proposition gives explicit expressions for $B$ and $\psi(s)$.

Corollary (of Proposition 4 and inequality (43)). There exist positive constants $c$ and $C$ such that

$$
c \leq e^{-\delta s} N(s) \leq C \quad \text { for } s \geq 0 .
$$

\section{Proof of the main results: the final step}

Choose $c_{k}=-\ln A_{k}, \quad k=1, \ldots, m$. Then, taking (42) in account, (37) and (41) become

$$
n\left(K \bar{A}(\mathcal{S}, \mathbf{p}) e^{-s}, T_{\mu}\right) \leq N(s) \leq n\left(C e^{-s}, T_{\mu}\right) .
$$

The conclusion of Theorems 1 and 2 immediately follows from the Corollary.

\section{Discussion}

1) The case $d=1$ was analyzed in [SVe]. Here $n\left(t, T_{\mu}\right)$ itself satisfies an equation similar to (44). As a result, more precise conclusions were obtained.

2) The Hausdorff dimension of $\mu$ equals

$$
\beta=\left(\sum_{k=1}^{m} p_{k} \ln p_{k}\right) /\left(\sum_{k=1}^{m} p_{k} \ln h_{k}\right)
$$

(see [St1]). The Hausdorff dimension $\alpha$ of $\mathcal{C}(\mathcal{S})$ is determined by the equation $\sum_{k=1}^{m} h_{k}^{\alpha}=1$ (see $[\mathrm{Hu}]$ ). Always $\beta \leq \alpha$, and the equality takes place if and only if $p_{k}=h_{k}^{\alpha}, \quad k=1, \ldots, m$. These $p_{k}$ 's are called "natural weights".

The exponent $\delta$ meets the following conditions:

$$
\begin{gathered}
\frac{\beta}{\beta+1} \leq \delta \leq \frac{\alpha}{\alpha+1} \quad \text { for } d=1 \quad(\text { see }[\mathrm{SVe}]), \\
\delta=1 \quad \text { for } d=2, \\
\text { (49) } \frac{\alpha}{\alpha-(d-2)} \leq \frac{\beta}{\beta-(d-2)} \leq \delta \quad \text { for } d \geq 3
\end{gathered}
$$


(the proof of (49) is similar to the proof of (48) given in [SVe]). If $d \geq 3$, the value of $\delta$ can be arbitrarily large. The equalities in (48), (49) take place only in the case of natural weights.

3) For the natural weights, $\mu(\mathcal{S}, \mathbf{p})$ is nothing but the restriction on $\mathcal{C}(\mathcal{S})$ of the Hausdorff measure of dimension $\alpha$. In this case the sufficiency part of Proposition 1 can be derived from Theorem 1 of Chapter 7 of [JWal].

4) If $d=2$, the only self-similar measures for which (12) fails, are deltameasures supported at an interior point of $\Omega$; here $m=1$.

5) The sign "=" in the inequality $\sum_{k=1}^{m} h_{k}^{d} \leq 1$ is rather rare. It corresponds to the case when $S_{k}(\Omega), \quad k=1, \ldots, m$, constitute a tiling of $\Omega$ by a family of subdomains similar to $\Omega$. If, in addition, $p_{k}$ are the natural weights, $\mu(\mathcal{S}, \mathbf{p})$ is nothing but the $d$-dimensional Lebesgue measure on $\Omega$. Here, clearly, $\delta=\frac{d}{2}$.

6 ) In the results of Section 3 , the spaces $H_{0}^{1}(X)$ and $H^{1}(X)$ endowed with the standard metric of $H^{1}$, can be taken instead of $L_{2}^{1}(X)$ and $L_{2}^{1}(X)$. The minor changes needed in the assumptions are evident. These changes correspond to the passage from (1) to the equation

$$
\lambda\left(\int_{X}(\nabla u \cdot \nabla \bar{v}) d x+\int_{X} u \bar{v} d x\right)=\int_{\bar{X}} u \bar{v} d \mu, \quad \text { any } v \in \mathcal{V} .
$$

The estimates (31) and (32) remain valid for this modified equation.

7) In the case $d=1$, it was proven in [Bo] that for any finite singular $\mu$ one has for the problem (1)

$$
n(t)=o\left(t^{-\frac{1}{2}}\right)
$$

(instead of $O\left(t^{-\frac{1}{2}}\right)$, given for $d=1$ by (3)). [Bo] contains also the first attempt to specify " $O$ " in (50), provided $\mu$ meets some additional restrictions. However, for self-similar $\mu$ the estimates obtained in [Bo] turn out to be not sharp. As was mentioned in Section 1, the estimate (5) for $d=1$ was first obtained in $[\mathrm{Fu}]$ with the help of another approach.

8) The equation (30) for $\delta$ has some resemblance with the equation for the so called spectral dimension $d_{S}$ of a "fractal object" (whose description includes a self-similar set endowed with a self-similar measure and with the so called harmonic structure; see [KLa]). The exponent $d_{S}$ appears in connection with the spectral analysis of the Laplacian on a fractal. This Laplacian and also the spectral dimension $d_{S}$ are determined by a fractal object and formally do not depend on the embedding into $\mathbb{R}^{d}$ (though usually the specific harmonic structure involved is implied by this embedding). 
On the contrary, in our type of problems the definition of the operators $T_{\mu}$ depends on this embedding.

\section{Acknowledgement}

We are obliged to V. Maz'ya from whom we learned about the statement given in Section 3 as Lemma 1. The proof adduced also belongs to V. Maz'ya. We are grateful to him for the permission to include this material into our text.

\section{References}

[BS] M. Birman and M. Solomyak, Quantitative analysis in Sobolev imbedding theorems and applications to spectral theory, Tenth Math. Summer School (Katsiveli/Nalchik, 1972), Izdanie Inst. Mat. Akad. Nauk Ukrain. SSR, Kiev (1974), 5-189 (in Russian); English transl. in Amer. Math. Soc. Transl. 114 (2) (1980).

[Bo] V. Borzov, Quantitative characteristics of singular measures, Problemy Mat. Fiz., vyp. 4, Leningrad (1970), 42-47 (in Russian); English transl. in Topics in Math. Phys., 4, Plenum Press, NY (1971).

[Fa] K. J. Falconer, The geometry of fractal sets, Cambridge University Press, 1985.

[Fe] W. Feller, An introduction to the probability theory and its applications, Vol. II, John Wiley \& Sons, Inc., New York-London-Sydney-Toronto, 1971.

[Fu] T. Fujita, A fractional dimension, self-similarity and a generalized diffusion operator, Taniguchi Symp. PMMP Katata (1985), 83-90.

[Hu] J. E. Hutchinson, Fractals and self-similarity, Indiana Univ. Math. J. 30 (1981), $713-747$.

[JWal] A. Jonsson and H. Wallin, Function spaces on subsets of $\mathbb{R}^{n}$, Mathematical Reports, Vol. 2, Part 1, J. Dieudonné (editor-in-chief), J. Peetre (issue editor), Harwood Academic Publishers, Chur-London-Paris-Utrecht-New York, 1984.

[KLa] J. Kigami and M. L. Lapidus, Weyl's problem for the spectral distribution of Laplacians on p.c.f. self-similar fractals, Commun. Math. Phys. 158 (1993), 93-125.

[La] M. L. Lapidus, Vibrations of fractal drums, the Riemann hypothesis, waves in fractal media and the Weyl-Berry conjecture, Ordinary and partial differential equations, Vol. 4, B. D. Sleeman and R. J. Jarvis (editors), Pitman Research Notes in Mathematics 289 (1993), 126-209.

[LauW] K.-S. Lau and J. Wang, Mean quadratic variations and Fourier asymptotics of self-similar measures, Monat. Math. 115 (1993), 99-132.

[LeVa] M. Levitin and D. Vassiliev, Spectral asymptotics, renewal theorem and the Berry conjecture for a class of fractals (1994), preprint. 
[M] V. Maz'ya, Sobolev spaces, Springer-Verlag, Berlin-Heidelberg-New YorkTokyo, 1985.

[NS] K. Naimark and M. Solomyak, On the eigenvalue behaviour for a class of operators related to self-similar measures on $\mathbb{R}^{d}$, C. R. Acad. Sci 319 (I) (1994), $837-842$.

[SVe] M. Solomyak and E. Verbitsky, On a spectral problem related to self-similar measures, Bull. of London Math. Soc. (to appear).

[Ste] E. M. Stein, Singular integrals and differentiability properties of functions, Princeton University Press, Princeton, NJ, 1970.

[St1] R. S. Strichartz, Self-similar measures and their Fourier transforms I, Indiana Univ. Math. J. 39 (1990), 797-817.

[St2] _ Self-similarity in harmonic analysis, preprint.

[Wo] M. Woodroofe, Nonlinear renewal theory in sequential analysis, SIAM, Philadelphia, 1982.

Department of Theoretical Mathematics, The Weizmann Institute of SciENCE, REHOVOT 76100 , ISRAEL

E-mail address: naimark@wisdom.weizmann.ac.il

Department of Theoretical Mathematics, The Weizmann Institute of SciENCE, REHOVOT 76100 , ISRAEL

E-mail address: solom@wisdom.weizmann.ac.il 